\section{Review Article}

\section{Check for updates}

\section{OPEN ACCESS}

\section{Received: Jun 7, 2017}

Accepted: Jun 23, 2017

\section{Correspondence to} Gianlorenzo Dionigi

Division of General Surgery, Research Center for Endocrine Surgery, Department of Human Pathology in Adulthood and Childhood "G. Barresi", University of Insubria and University Hospital Policlinico G. Martino, University of Messina, Via C. Valeria Gazzi, Messina 98125, Italy.

Tel: +39-3495115877

E-mail: gianlorenzo.dionigi@gmail.com

Copyright $($ 2017. Korean Association of Thyroid and Endocrine Surgeons; KATES This is an Open Access article distributed under the terms of the Creative Commons Attribution Non-Commercial License (https:// creativecommons.org/licenses/by-nc/4.0/).

ORCID iDs

Young Jun Chai (D)

https://orcid.org/0000-0001-8830-3433

No Author Contributions

Conceptualization: name. Data curation: name. Formal analysis: name. Funding acquisition: name. Investigation: name. Methodology: name. Project administration: name. Resources: name. Software: name. Supervision: name. Validation: name. Visualization: name. Writing - original draft: name. Writing - review \& editing: name.

\title{
Standards for Intraoperative Neuromonitoring in Thyroid Operations
}

\author{
Hoon Yub Kim', Xiaoli Liư ${ }^{2}$, Young Jun Chai ${ }^{(1)}{ }^{3}$, Ralph Tufano ${ }^{4}$, Henning Dralle, \\ Gianlorenzo Dionigi ${ }^{6}$, the Korean Intraoperative Neural Monitoring Society (KINMoS)
}

'KUMC Thyroid Center, Korea University Anam Hospital, Seoul, Korea

${ }^{2}$ Division of Thyroid Surgery, Jilin Provincial Key Laboratory of Surgical Translational Medicine, China Japan Union Hospital of Jilin University, Changchun, China

${ }^{3}$ Department of Surgery, Seoul Metropolitan Government-Seoul National University Boramae Medical Center, Seoul, Korea

${ }^{4}$ Department of Otolaryngology-Head and Neck Surgery, The Johns Hopkins University School of Medicine, Baltimore, MD, USA

${ }^{5}$ Department of General, Visceral, and Transplantation Surgery, University Hospital Essen, Essen, Germany ${ }^{6}$ Division of General Surgery, Research Center for Endocrine Surgery, Department of Surgical Sciences and Human Pathology, University of Insubria, Varese, Italy

\section{ABSTRACT}

After the introduction of intraoperative neural monitoring (IONM) of the recurrent laryngeal nerve (RLN) in clinical thyroid practice almost 16 years ago, the procedure has expanded rapidly with an area-wide spread in Asia, Europe, and USA. While the visual nerve presentation with the eye or the magnifying glass technique is capable of assessing the anatomical continuity of the RLN, IONM additionally allows a functional analysis that has a high correlation, i.e., prediction of postoperative vocal motility. Although the predictive value of the IONM is much higher $(>97 \%)$ in the case of an intact signal than in the case of a signal failure ( $40 \%-70 \%)$, the prediction is also unequally higher than the visual-anatomical assessment of the nerve. Thus, IONM can be used as a basis for an intraoperative decisionmaking of a 1-side or 2-side procedure to avoid bilateral RLN palsy in a bilateral procedure. A precondition for the safe application of IONM is the perfect knowledge of the technology and technique, the routine execution of preoperative and postoperative laryngoscopy, the strict standardization of the neurostimulation (electromyography documentation of the vagal nerve stimulation before and after resection), and an adequate management of technically or operationally caused incidents (i.e., systematic application of troubleshooting algorithms). The following review provides a synopsis of the experiences of the Korean Intraoperative Neural Monitoring Society (KINMoS) for the correct use of IONM.

Keywords: Thyroid gland; General surgery; Recurrent laryngeal nerve; Nerve injury; Thyroidectomy

\section{INTRODUCTION}

Since the beginning of thyroid surgery, the surgical morbidity has been the focus of discussions [1-5]. Injury to the recurrent laryngeal nerve (RLN) is the leading cause of vocal cord paralysis associated with hoarseness, impaired vocal register, dysphonia, dysphagia, and aspiration $[4,5]$. After decades of debates about the prognosis of the laryngeal nerves, the conviction that thyroid gland resections in nerve proximity, i.e., total resection, visual nerve 
Conflict of Interest

No potential conflict of interest relevant to this article was reported. identification, exposure, and presentation, are the best possible condition "gold standards" management for the RLN (Table 1) [6-13].

Previous studies reported a $0.4 \%-3.9 \%$ incidence of temporary RLN paralysis and a $0 \%-$ $3.6 \%$ incidence of permanent RLN paralysis after thyroidectomy [6-13].

Intraoperative neuromonitoring (IONM) has been proposed as an adjunct to standard visual identification of the laryngeal nerves during thyroid surgery [14]. Technical advances currently allow accurate and noninvasive monitoring of the nerves [14]. With the availability of IONM, a new element has been introduced into the RLN management: the electromyographic (EMG) function of the nerve [14]. However, in order to enhance the outcomes of IONM on postoperative vocal cord function, the following prerequisites must be fulfilled:

1. Standardization of the technique, and specific experience and training with IONM device are essential for optimal use.

2. Correct functioning of the IONM equipment with regard to nerve stimulation "stimulation site" and the signal recording and reproducing "recording site."

3. Intact and sufficient nerve conduction, including transfer into an adequate muscle action of the stimulated vocalis muscle.

4. Exclusion of pre-existing or endotracheal-induced non-nervous disorders of the vocal cord mobility.

So far, the results on the use of IONM have been discordant. For example, an IONM signal failure with postoperatively intact voice mobility, has been reported "right" on the one hand (i.e., loss of the nerve function with recovery of the nerve conduction at the time of the postoperative laryngeal examination, and "wrong" on the other hand (i.e., errors in the "stimulation" or "recording pattern" for intra- and postoperatively intact nerve function. This has led to an intensive discussion about the application of the IONM technology and its suitability for greater safety in the prevention of nerve damage $[14,15]$.

In addition, it is understandable that both 1 ) a very strict standardization in the use of IONM and 2) a suitable "troubleshooting" in case of disturbances, i.e., signal failure, are necessary, while 3) the knowledge of the clinical larynx function (preoperative and postoperative laryngoscopy) must be evaluated, because the consequences of an intraoperative alteration of the primarily intended resection dimension (e.g., only unilateral rather than bilateral resection, "staged procedure") and the necessary medico-legal expert assessment of an individual case can only be carried out correctly if the above mentioned conditions are met [15].

Table 1. Neural monitoring enhances the "gold standards" for RLN management in thyroid surgery

\footnotetext{
- Extensive knowledge of RLN anatomy

- Visual identification of RLN

- Exposure of RLN

- Experience and training

- Pre- and postoperative laryngoscopy

$\mathrm{RLN}=$ recurrent laryngeal nerve.
} 
In addition to the technical aspects of RLN monitoring, it is currently clear that the available methods of intermittent or continuous nerve stimulation prevent the occurrence of RLN paresis in case of disturbances like traction or compression, but not RLN stress as a result of acute nerve injuries as in the case of a coagulation, ligation, or section [16-22].

Currently, it is clear from both experimental and clinical studies that the peculiarities of the nerve physiology of the RLN contain a gradual diminishing nervous function, e.g., during prolonged traction on the laryngeal nerve, with increased latency and decreased amplitude profile. Nevertheless, it is still not clear when and at what thresholds of amplitude and latency do these become irreversible [14,16-22].

\section{STANDARDIZATION OF RLN MONITORING}

Few surgeons have received formal training in using IONM. The Korean Intraoperative Neural Monitoring Society (KINMoS) offers and promotes continuous knowledge and training for the correct, systematic, and standard approach to IONM technique.

The standardization of intraoperative RLN monitoring is based on the following basic requirements (Table 2) [15]:

1. Preoperative and postoperative laryngoscopy and intraoperative EMG are inseparable units. The preoperative intraoperative EMG findings cannot be assessed without preoperative laryngoscopy findings. Similarly, the postoperative intraoperative EMG findings are also not possible to correlate without the postoperative laryngoscopy findings interpretation. Thus, preoperative and postoperative laryngeal examinations are a prerequisite for the clinical use of the IONM method. A preoperative laryngeal detection of RLN palsy must be interpreted with caution, because, in spite of paralyzed vocal motility, a nerve can still present with a positive EMG signal. Therefore, for the IONM, the following explanations refer to the situation of a preoperatively intact vocal laryngeal mobility, but not to the situation of a pre-existing vocal cord paresis.

2. The routine intraoperative check of the functional status of the IONM system to prove the concordance of preoperative vocal cord movement and neurostimulation. The correctness evaluation method requires nerve stimulation outside the potential operative area of the RLN. For this, the ipsilateral vagal stimulation is the safest. The results of the largest evaluation study carried out for this purpose clearly demonstrated the importance of this approach, i.e., pre-excision vagal nerve (VN) stimulation (Fig. 1) $[15,23]$.

Table 2. The following standardized steps are required for the proper use of intraoperative RLN monitoring in clinical thyroid practice

- Preoperative assessment of the vocal cord function by laryngoscopy (L1)

- Use of modern audio and graphic IONM monitor software with documented latency and ampliude profile for the safe differentiation of artifact signals

- Verify meticulously the correct equipment installation, including placing the neutral and recording electrodes

- Use of short-acting muscle relaxants only during anesthesia initiation, renouncing this during the phase of the IONM

- Nerve stimulation with current amplitudes of 1-2 mA above the threshold current (0.3-0.8 mA)

- Ipsilateral stimulation of the vagus nerve with 1-2mA (above the threshold current intensity) and EMG recording before the start of the resection (V1)

- Visual presentation and ipsilateral stimulation of the RLN (R1)

- Post-resection ipsilateral stimulation of the VN (V2)

- Post-resection ipsilateral stimulation of the RLN (R2)

- Dispense the resection of the second side in the case of primarily planned bilateral procedure and loss of signal on the first side

- Postoperative assessment of the vocal cord function by laryngoscopy (L2)

$\mathrm{RLN}=$ recurrent laryngeal nerve; IONM = intraoperative neuromonitoring; $\mathrm{EMG}=$ electromyographic; $\mathrm{VN}=\mathrm{vagal}$ nerve. 


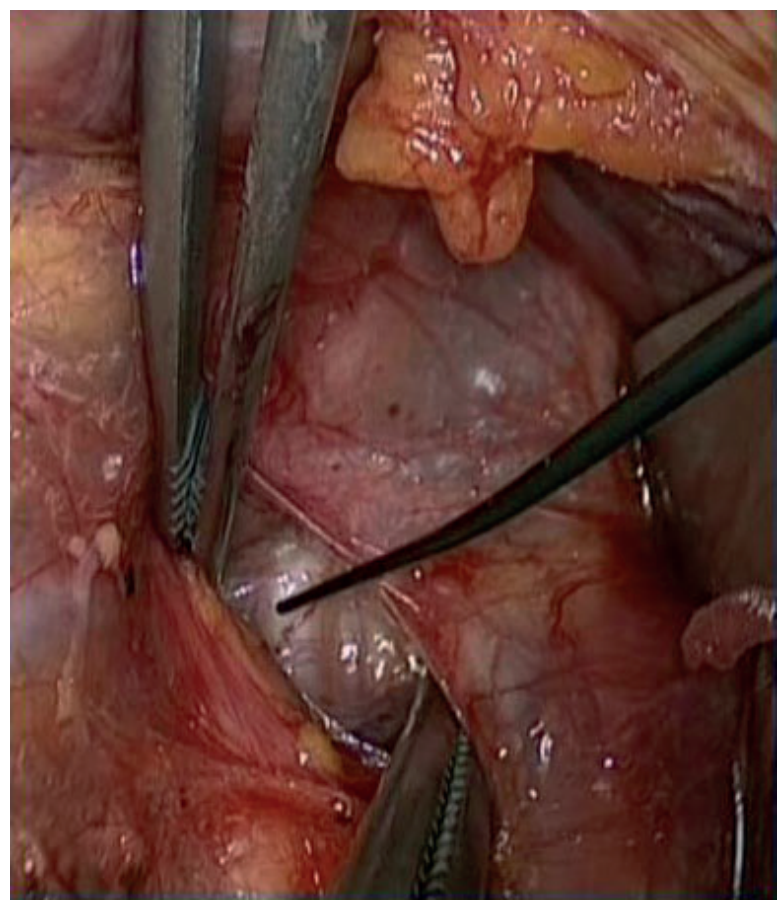

Fig. 1. Pre-dissection VN stimulation (V1). $\mathrm{VN}=$ vagal nerve.

3. To achieve adequate nerve stimulation, the RLN and the VN should be stimulated beyond the threshold current of $0.3-0.8 \mathrm{~mA}$. The optimal current strength for nerve stimulation is 1-2 mA. There is no further improvement and achievement of the muscle response of the vocalis muscle with a higher current strength [24].

4. Anesthesia management during intraoperative neuromonitoring does not require the use of muscle relaxants during the neuromonitoring phase, since these can impair the vocalis muscle response leading to intraoperative misinterpretations [25]. During induction anesthesia, the use of shortacting muscle relaxants (succinylcholine [Anectine ${ }^{\circledR}$; Sandoz Inc., Princeton, NJ, USA] 2.0-2.5 mg/ kg or rocuronium [Zemuron ${ }^{\mathrm{T}}$, Merck Canada Inc., Kirkland, Canada] or atracurium [Tracrium ${ }^{\circledR}$; GlaxoSmithkline Pharmaceuticals Ltd., Mumbai, India] $0.5 \mathrm{mg} / \mathrm{kg}$ ) is recommended in order to ensure adequate muscle activity when using nerve stimulation [25].

5. The correct IONM apparatus requires adequate placement of the cable connections, neutral electrodes, and EMG signal electrodes (i.e., needle electrodes or EMG endotracheal tube electrodes) (Fig. 2) [24]. When the needle electrodes are used, they must be inserted into the vocalis muscle separately for each of the operated sides $[15,24]$. When EMG endotracheal tube electrodes are used, it is necessary to ensure that the cuff of the tube is located below the glottal plane and the signal derivation electrodes above, i.e., in the glottal plane of the vocal folds, and not rotated after the patient has been positioned (Fig. 3) [24]. The proper contact of the EMG tube with the vocal cords is indicated by the following verification test: 1 ) impedance values readable on the IONM monitor less than $5 \mathrm{k} \Omega$ per electrode; 2) repeat laryngeal examination after the patient's head extension to check possible tube displacement; 3) respiratory variation; 4) tap test; and 5) intraoperative V1 above $500 \mathrm{mcV}$ (Table 3) [24]. 


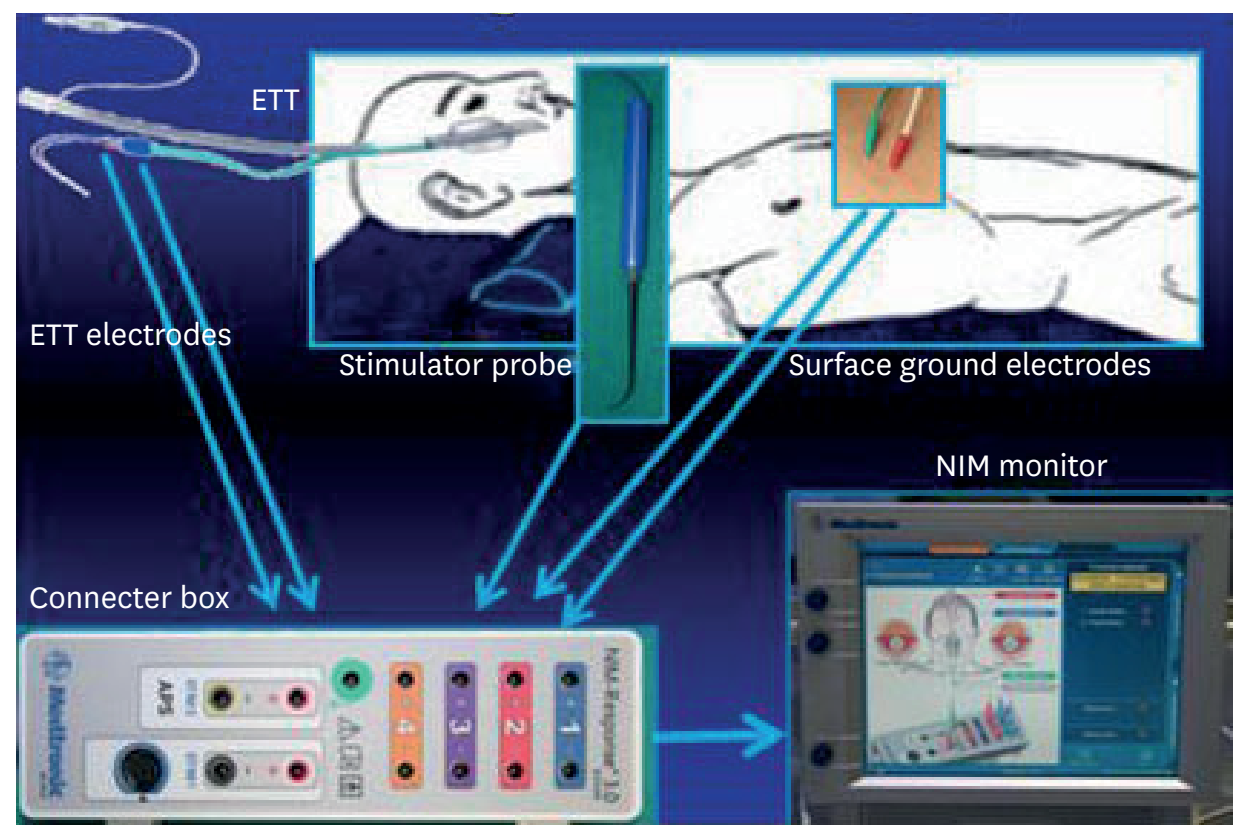

Fig. 2. Patient and EMG endotracheal tube scheme for IONM.

$E M G$ = electromyographic; IONM = intraoperative neuromonitoring; $E T T$ = endotracheal tube; $\mathrm{NIM}$ = nerve integrity monitor.

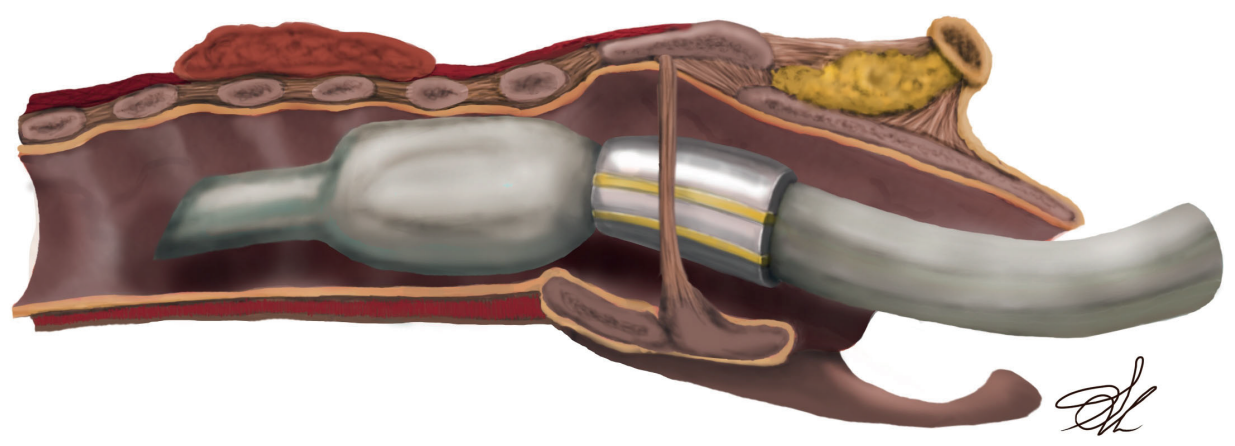

Fig. 3. When EMG endotracheal tube electrodes are used, it is to be ensured that the signal surface derivation electrodes are positioned in the glottal plane of the vocal folds and are not rotated after the patient has been stored. $E M G$ = electromyographic.

Table 3. Verification test for proper contact of the EMG tube with the vocal cords

\begin{tabular}{|c|c|}
\hline \multirow[t]{4}{*}{ Preoperatively } & $\begin{array}{l}\text { 1) The impedance values readable on the IONM monitor, it should be less than } 5 \mathrm{k} \Omega \text { per } \\
\text { electrode }\end{array}$ \\
\hline & $\begin{array}{l}\text { 2) Repeat laryngeal examination after patient head extension to check possible tube } \\
\text { desplacemnet }\end{array}$ \\
\hline & 3) Respiratory variation \\
\hline & 4) Tap test \\
\hline Intraoperatively & $\begin{array}{l}\text { 5) Achieve intraoperative } \mathrm{V} 1 \text { above } 500 \mathrm{mcV} \\
\text { 6) All above }\end{array}$ \\
\hline
\end{tabular}

EMG = electromyographic; IONM = intraoperative neuromonitoring. 


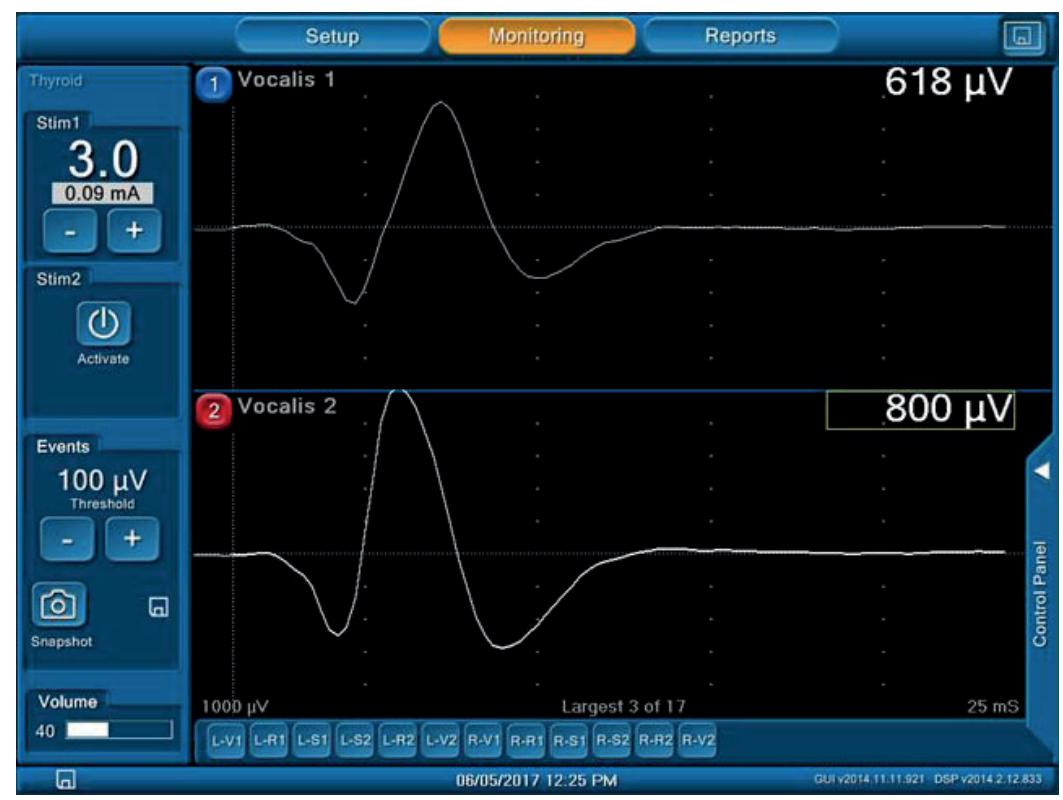

Fig. 4. The use of audio and EMG signal equipment is recommended to enhance documentation of the stimulation EMG with quantification of latency and amplitude.

$E M G=$ electromyographic.

Table 4. Unique features of audio and graphic IONM monitors for RLN monitoring

- Amplitude and latency profile recording

- Documentation

- Quantification

- Storage

- Differentiation between signal and artifact

- Forensic

- Research

- To justify surgical deliberations

- Record review and discussion at end of surgery

IONM = intraoperative neuromonitoring; RLN = recurrent laryngeal nerve.

6. The unequivocal determination of a regular nerve conduction and consecutive muscle action of the ipsilateral vocalis muscle requires qualitative and quantitative EMG recordings with amplitude and latency by the IONM device, since the sole signal sound reproduction cannot reliably distinguish between an artifact signal and a regular muscular action potential [15]. The use of modern audio and EMG signal equipment is recommended to enhance, if possible, the documentation of the stimulation EMG (Fig. 4) [15]. Not only does this allow a reliable detection of the muscle potential but the stimulation of the RLN or the VN provides a latency of the recurrent nerve and the vagus nerve significantly different from one another due to the different nerve lengths (Table 4) [15]. The different nerve lengths of the left and right nasal vagus also allow the respective EMG to be assigned to the right or left VN during stimulation of the vagus nerve for a secure lateral allocation $[15,24]$.

\section{TROUBLESHOOTING AND CONSEQUENCES FOR INTRAOPERATIVE SIGNAL LOSS}

The definition of an intraoperative EMG signal loss ("loss of signal", LOS) presupposes that the initial signal response was correct (Table 5). If the intraoperative signal fails or the EMG 
Table 5. Definition of loss of EMG signal

- Normal vocal cord movement at preoperative laryngeal examination (L1)

- Initial satisfactory EMG signal (V1 $>500 \mathrm{mcV}$ )

- No EMG response with stimulation at 1-2 mA

- Low response $<100 \mathrm{mcV}$ with stimulation at 1-2 mA

- No laryngeal twitch

- Troubleshooting algorithm applied systematically

EMG = electromyographic.

drops below $100 \mu \mathrm{V}$ with a primary intact signal and adequate stimulation with $1-2 \mathrm{~mA}$, a LOS must be assumed.

The IONM troubleshooting algorithm consists of the following steps in this sequence (Fig. 5): 1) Exclusion of anesthesia-induced neuromuscular blockade, device-technical disturbances, dislocation of the neutral electrode or of the cable connections, tube dislocation, or dislocation of the surface electrodes. 2) Stimulation of the ipsilateral RLN before its entry into the larynx and stimulation of the ipsilateral $\mathrm{VN}$; this is subsequently followed by verifying the stimulation

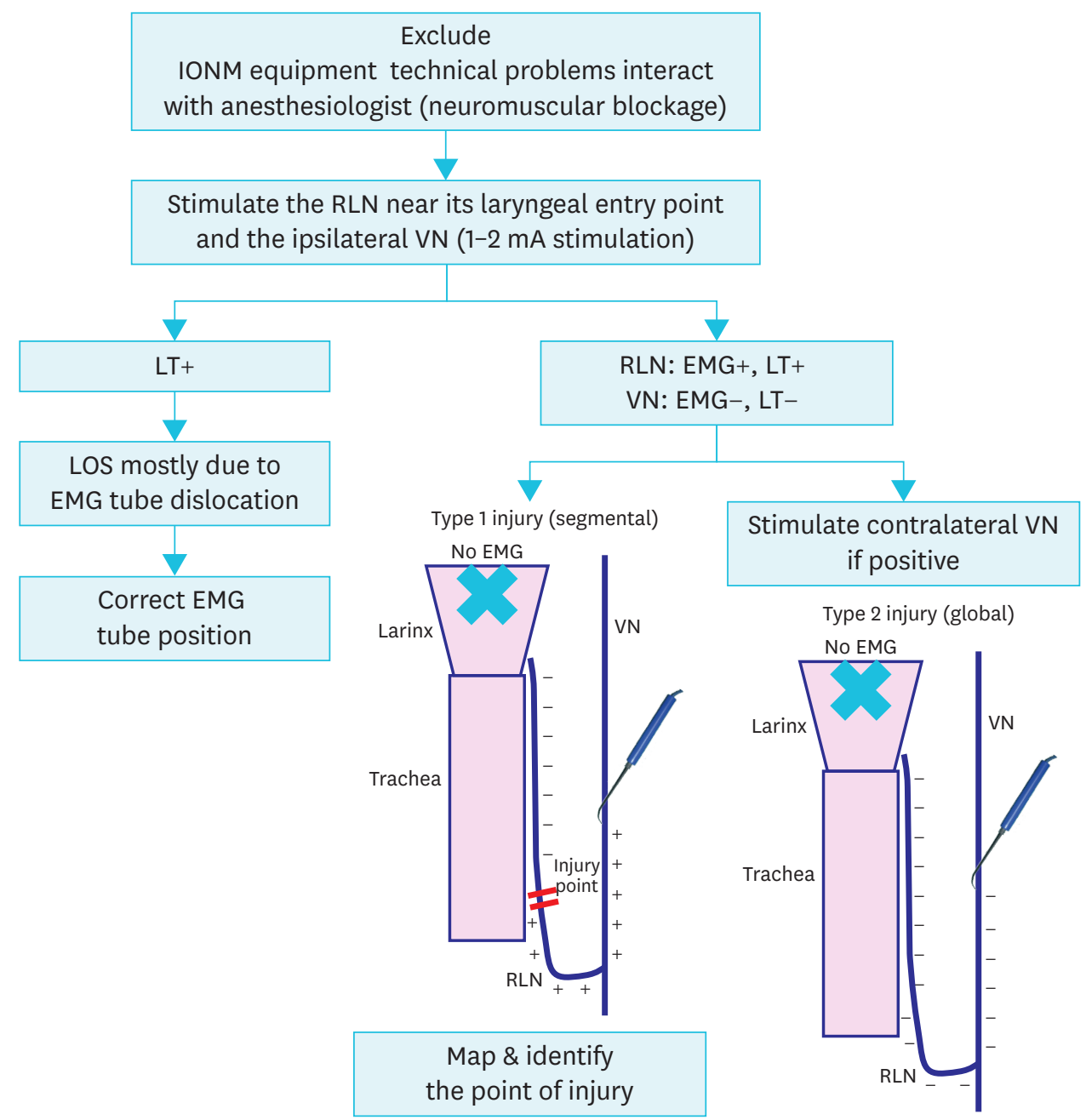

Fig. 5. Troubleshooting algorithm in case of loss of EMG signal. $\mathrm{EMG}=$ electromyographic. 


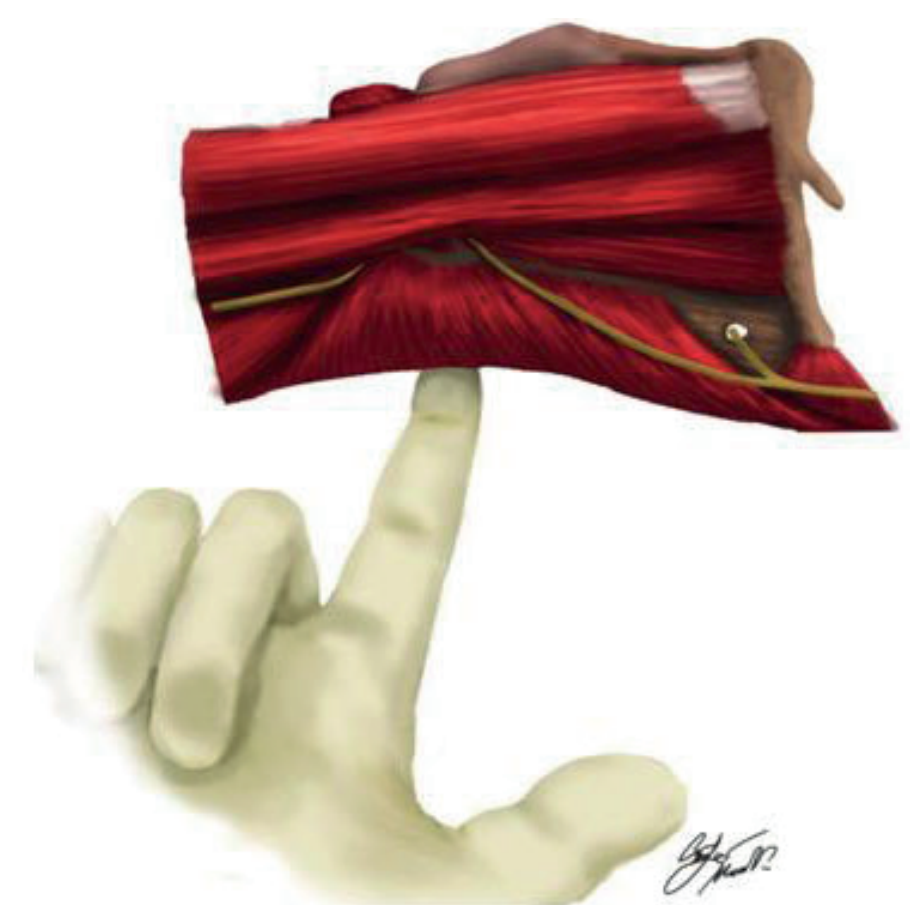

Fig. 6. Laryngeal twitch.

response by means of EMG and possibly palpation of the dorsal larynx wall (laryngeal twitch) as a positive sign of an equilateral muscle action of the vocalis muscle (Fig. 6) [12].

Depending on the stimulation site and the stimulation response, the following conclusions can be drawn with regard to the main disorders of the ipsilateral RLN (Fig. 5):

1. Positive laryngeal twitch independent of the location of the stimulation: consider error of the "recording site," i.e., EMG endotracheal tube dislocation or needle dislocation. This necessitates a correction of the tube or needle electrode position in case of the use of needle electrodes.

2. A normal EMG and/or positive laryngeal twitch during stimulation of the RLN at the point of entry into the larynx, but a lack of an EMG response or missing laryngeal twitch when stimulating the vagus nerve: consider a real loss of function of the ipsilateral RLN. In this type of nerve disorder, the location of the nerve lesion can usually be localized by "mapping" (type 1, point/segmental injury, Fig. 7).

3. A lack of an EMG response and a missing laryngeal twitch regardless of the location of the stimulation (RLN or vagus nerve) means a diffuse nervous disorder (type-2 global injury, Fig. 7), assuming correct stimulation and exclusion of the causes mentioned above.

4. If there is any uncertainty regarding the function of the equipment, tube position, or position of the needle electrode, the contralateral VN should be stimulated. When the nerve function of the contralateral VN is intact, a disturbed nerve function can be assumed in the absence of a stimulation response from the side in question.

Discordant findings, i.e., neuromonitoring signal and EMG deviations from the postoperative vocal cord mobility, are extremely rare $(<3 \%)$ with an intact neuromonitoring signal and EMG. In contrast, in the case of intraoperative signal failure, they are observed in about $30 \%-60 \%$ 
Type 1 injury (segmental)

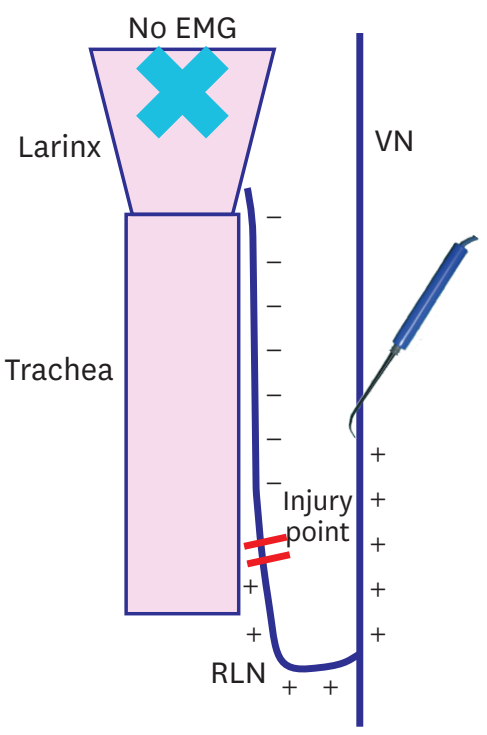

Type 2 injury (global)

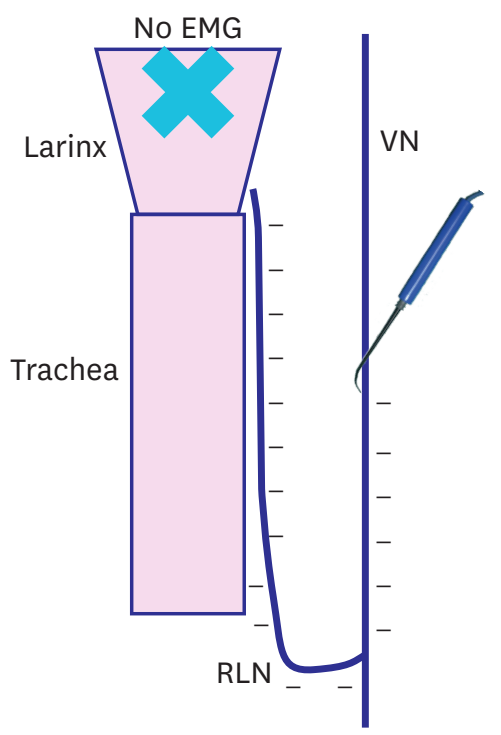

Fig. 7. Type 1 and type 2 injuries.

of the cases. Based on this, the surprising finding of a postoperative RLN paresis is extremely rare with intraoperatively intact signal and EMG. In the case of intraoperative signal failure, however, postoperative RLN paresis is expected at only $30 \%-60 \%$.

Due to the significantly higher predictive significance of intraoperative EMG with respect to the postoperative vocal cords compared to the visual-anatomical assessment alone after resection of a signal failure first side, if the first side of a planned 2-sided resection is concerned, it should be not recommended to remove the contralateral side in order to avoid the risk of bilateral vocal cord palsy. In bilateral benign goiter and most low-risk carcinomas, there is generally no need for resection of the contralateral side in case of intraoperative signal loss on the first side. The laryngoscopy on the first postoperative day reveals whether or not an RLN palsy is present on the disturbed side; if not, the resection of the second side can be made immediately or, depending on the urgency of the situation, at a later date [16-29].

\section{CONCLUSIONS AND ASSESSMENTS IN CASE OF RLN} INJURY

Most of the multicentric retrospective studies and prospective randomized studies have reported a decrease in the RLN palsy rate using IONM vs. the "gold standard" of the visual nerve identification only, but the difference between the two techniques was not statistically significant $[2,30,31]$.

An low surgical experience has been associated with a lower paresis rate when IONM is used $[30,31]$.

Since not only high risk interventions but also atypical RLN variants and locations are associated with a demonstrable increase in RLN paresis risk in primary procedures, it is to be assumed that neuromonitoring is also advantageous in such situations due to a better RLN prognosis [32]. 
In recent years, the expert assessment of intraoperative neuromonitoring has repeatedly led to the assessment of RLN paresis in cases of damage; IONM guidelines for the proper use of intraoperative neuromonitoring are now published [24].

The position of the surgical expert in such cases is not easy because the correct assessment of the individual case requires not only experience with neuromonitoring but also, at least, a certain degree of traceability of the use of this method in the given case, i.e., a corresponding EMG documentation of neurostimulation in order to draw justifiable conclusions from a surgical perspective.

Currently, the following observations can be made for the expert assessment of intraoperative neuromonitoring on the basis of the above-described advantages and limitations of this method:

1. When intraoperative neuromonitoring is used, the following points should be considered: 1) compliance with the standards set-up (Table 2) and 2) systematic use of troubleshooting algorithms in the case of intraoperative signal loss (Fig. 5). Both points are essential for a correct interpretation of intraoperative EMG signals and, in the case of intraoperative signal loss, to draw appropriate conclusions.

2. Since the signal tone alone does not differ sufficiently reliably between artifact and regular muscle action potential, the simultaneous EMG recording alone represents the "gold standard" of the nerve function assessment. The only and best documentation currently available for an expert assessment of the intraoperative nerve function state is the EMG expression of ipsilateral vagal stimulation before and after resection.

3. The sensitivity of intraoperative neuromonitoring with regard to the assessment that postoperative RLN paresis follows an intraoperative signal failure is still unsatisfactory. Nevertheless, the intraoperative signal failure represents the best currently available technique, significantly superior risk criterion vs. the only anatomical-visual assessment for the prediction of a postoperative recurrence paresis. Thus, in case of a signal loss on the first operated side within an intended bilateral procedure the resection of the second side should be dispensed in the same session until the recovery of the nerve in order to avoid the risk of bilateral recurrent palsy. The reason for the premature bilateral resection in this situation has to be linked to high-threshold conditions, since there are very few indications under which the resection of the contralateral side could not occur at a later time.

4. Preoperative patient information (informed consent) should adequately take into account this neuromonitoring-dependent procedural strategy by informing the patient that intraoperative signal failure of the first operated side is generally dispensed with a 2-step procedure for the sake of avoiding 2-way RLN paresis more safely.

\section{REFERENCES}

1. Dralle H, Sekulla C, Haerting J, Timmermann W, Neumann HJ, Kruse E, et al. Risk factors of paralysis and functional outcome after recurrent laryngeal nerve monitoring in thyroid surgery. Surgery 2004;136:1310-22. PUBMED | CROSSREF

2. Hermann M, Alk G, Roka R, Glaser K, Freissmuth M. Laryngeal recurrent nerve injury in surgery for benign thyroid diseases: effect of nerve dissection and impact of individual surgeon in more than 27,000 nerves at risk. Ann Surg 2002;235:261-8. PUBMED | CROSSREF 
3. Cho I, Jo MG, Choi SW, Jang JY, Wang SG, Cha W. Some posterior branches of extralaryngeal recurrent laryngeal nerves have motor fibers. Laryngoscope, in press 2017. PUBMED | CROSSREF

4. Baek SK, Lee K, Oh D, Kang SH, Kwon SY, Woo JS, et al. Efficiency of intraoperative neuromonitoring on voice outcomes after thyroid surgery. Auris Nasus Larynx 2017;44:583-9. PUBMED | CROSSREF

5. Cha W, Cho I, Jang JY, Cho JK, Wang SG, Park JH. Supramaximal neurostimulation with laryngeal palpation to predict postoperative vocal fold mobility. Laryngoscope 2016;126:2863-8. PUBMED | CROSSREF

6. Wu CW, Hao M, Tian M, Dionigi G, Tufano RP, Kim HY, et al. Recurrent laryngeal nerve injury with incomplete loss of electromyography signal during monitored thyroidectomy-evaluation and outcome. Langenbecks Arch Surg 2017;402:691-9. PUBMED | CROSSREF

7. Lee MS, Lee UY, Lee JH, Han SH. Relative direction and position of recurrent laryngeal nerve for anatomical configuration. Surg Radiol Anat 2009;31:649-55. PUBMED | CROSSREF

8. Roh JL, Yoon YH, Park CI. Recurrent laryngeal nerve paralysis in patients with papillary thyroid carcinomas: evaluation and management of resulting vocal dysfunction. Am J Surg 2009;197:459-65. PUBMED | CROSSREF

9. Yun JS, Lee YS, Jung JJ, Nam KH, Chung WY, Chang HS, et al. The Zuckerkandl's tubercle: a useful anatomical landmark for detecting both the recurrent laryngeal nerve and the superior parathyroid during thyroid surgery. Endocr J 2008;55:925-30.

PUBMED | CROSSREF

10. Hong KH, Kim YK. Phonatory characteristics of patients undergoing thyroidectomy without laryngeal nerve injury. Otolaryngol Head Neck Surg 1997;117:399-404. PUBMED | CROSSREF

11. Lamadé W, Renz K, Willeke F, Klar E, Herfarth C. Effect of training on the incidence of nerve damage in thyroid surgery. Br J Surg 1999;86:388-91. PUBMED | CROSSREF

12. Randolph GW, Kobler JB, Wilkins J. Recurrent laryngeal nerve identification and assessment during thyroid surgery: laryngeal palpation. World J Surg 2004;28:755-60. PUBMED | CROSSREF

13. Sosa JA, Bowman HM, Tielsch JM, Powe NR, Gordon TA, Udelsman R. The importance of surgeon experience for clinical and economic outcomes from thyroidectomy. Ann Surg 1998;228:320-30. PUBMED | CROSSREF

14. Barczyński M, Konturek A, Cichoń S. Randomized clinical trial of visualization versus neuromonitoring of recurrent laryngeal nerves during thyroidectomy. Br J Surg 2009;96:240-6. PUBMED | CROSSREF

15. Dralle H, Lorenz K. Intraoperative neuromonitoring of thyroid gland operations: surgical standards and aspects of expert assessment. Chirurg 2010;81:612-9.

PUBMED | CROSSREF

16. Schneider R, Randolph GW, Barczynski M, Dionigi G, Wu CW, Chiang FY, et al. Continuous intraoperative neural monitoring of the recurrent nerves in thyroid surgery: a quantum leap in technology. Gland Surg 2016;5:607-16. PUBMED | CROSSREF

17. Schneider R, Sekulla C, Machens A, Lorenz K, Thanh PN, Dralle H. Dynamics of loss and recovery of the nerve monitoring signal during thyroidectomy predict early postoperative vocal fold function. Head Neck 2016;38 Suppl 1:E1144-51. PUBMED | CROSSREF

18. Phelan E, Schneider R, Lorenz K, Dralle H, Kamani D, Potenza A, et al. Continuous vagal IONM prevents recurrent laryngeal nerve paralysis by revealing initial EMG changes of impending neuropraxic injury: a prospective, multicenter study. Laryngoscope 2014;124:1498-505. PUBMED | CROSSREF

19. Schneider R, Bures C, Lorenz K, Dralle H, Freissmuth M, Hermann M. Evolution of nerve injury with unexpected EMG signal recovery in thyroid surgery using continuous intraoperative neuromonitoring World J Surg 2013;37:364-8. PUBMED | CROSSREF

20. Schneider R, Randolph GW, Sekulla C, Phelan E, Thanh PN, Bucher M, et al. Continuous intraoperative vagus nerve stimulation for identification of imminent recurrent laryngeal nerve injury. Head Neck 2013;35:1591-8. PUBMED | CROSSREF 
21. Schneider R, Sekulla C, Machens A, Lorenz K, Nguyen Thanh P, Dralle H. Postoperative vocal fold palsy in patients undergoing thyroid surgery with continuous or intermittent nerve monitoring. Br J Surg 2015;102:1380-7. PUBMED | CROSSREF

22. Dralle H, Sekulla C, Lorenz K, Nguyen Thanh P, Schneider R, Machens A. Loss of the nerve monitoring signal during bilateral thyroid surgery. Br J Surg 2012;99:1089-95. PUBMED | CROSSREF

23. Thomusch O, Sekulla C, Machens A, Neumann HJ, Timmermann W, Dralle H. Validity of intra-operative neuromonitoring signals in thyroid surgery. Langenbecks Arch Surg 2004;389:499-503. PUBMED | CROSSREF

24. Randolph GW, Dralle H, International Intraoperative Monitoring Study GroupAbdullah H, Barczynski $\mathrm{M}$, Bellantone R, et al. Electrophysiologic recurrent laryngeal nerve monitoring during thyroid and parathyroid surgery: international standards guideline statement. Laryngoscope 2011;121 Suppl 1:S1-16. PUBMED | CROSSREF

25. Marusch F, Hussock J, Haring G, Hachenberg T, Gastinger I. Influence of muscle relaxation on neuromonitoring of the recurrent laryngeal nerve during thyroid surgery. Br J Anaesth 2005;94:596-600. PUBMED | CROSSREF

26. Melin M, Schwarz K, Lammers BJ, Goretzki PE. IONM-guided goiter surgery leading to two-stage thyroidectomy--indication and results. Langenbecks Arch Surg 2013;398:411-8. PUBMED | CROSSREF

27. Périé S, Aït-Mansour A, Devos M, Sonji G, Baujat B, St Guily JL. Value of recurrent laryngeal nerve monitoring in the operative strategy during total thyroidectomy and parathyroidectomy. Eur Ann Otorhinolaryngol Head Neck Dis 2013;130:131-6. PUBMED | CROSSREF

28. Dionigi G, Frattini F. Staged thyroidectomy: time to consider intraoperative neuromonitoring as standard of care. Thyroid 2013;23:906-8. PUBMED | CROSSREF

29. Fontenot TE, Randolph GW, Setton TE, Alsaleh N, Kandil E. Does intraoperative nerve monitoring reliably aid in staging of total thyroidectomies? Laryngoscope 2015;125:2232-5. PUBMED | CROSSREF

30. Dralle H, Sekulla C, Haerting J, Timmermann W, Neumann HJ, Kruse E, et al. Risk factors of paralysis and functional outcome after recurrent laryngeal nerve monitoring in thyroid surgery. Surgery 2004;136:1310-22. PUBMED | CROSSREF

31. Dralle H, Sekulla C, Lorenz K, Brauckhoff M, Machens AGerman IONM Study Group. Intraoperative monitoring of the recurrent laryngeal nerve in thyroid surgery. World J Surg 2008;32:1358-66. PUBMED | CROSSREF

32. Casella C, Pata G, Nascimbeni R, Mittempergher F, Salerni B. Does extralaryngeal branching have an impact on the rate of postoperative transient or permanent recurrent laryngeal nerve palsy? World J Surg 2009;33:261-5.

PUBMED | CROSSREF 